\title{
Avaliação das denúncias de ineficácia terapêutica de medicamentos com resultados satisfatórios
}

Amanda da Silva Rio

Mestre em Vigilância Sanitária, INCQS/Fiocruz

Katia Christina Leandro

Doutora em Química, Puc-Rio

Letícia Kegele Lignani

Mestre em Química, UFRJ

Mychelle Alves Monteiro

Doutora em Engenharia de Processos Químicos e Bioquímicos, UFRJ 


\section{Resumo}

A administração de medicamentos ineficazes pode trazer consequências graves para a saúde do paciente, e no caso de medicamentos utilizados para tratar doenças potencialmente fatais pode colocar a vida em risco. Este estudo teve por objetivo avaliar as denúncias com suspeita de ineficácia terapêutica nas amostras de medicamentos com resultados satisfatórios analisadas no Instituto Nacional de Controle da Qualidade em Saúde (INCQS) no período de janeiro de 2000 a dezembro de 2016 e verificar as possíveis fontes de agravo à saúde pública. Do total de 1974 amostras de medicamentos provenientes de denúncias, 424 (21\%) foram identificadas com suspeita de ineficácia terapêutica, sendo $318(75 \%)$ com resultados analíticos satisfatórios. Dentre estes medicamentos satisfatórios, 35 (11\%) refere-se a medicamentos de referência, 68 (21\%) a genéricos e $215(68 \%)$ a similares. Os princípios ativos que apresentaram maiores notificações, mas com resultados satisfatórios foram captopril, maleato de enalapril, carbamazepina e cloridrato de bupivacaína. Todos constam na literatura como fármacos que podem apresentar polimorfos, além disso, captopril, maleato de enalapril e cloridrato de bupivacaína apresentam quiralidade. Entretanto, os ensaios analíticos preconizados nas Farmacopeias nem sempre são suficientes para identificar possíveis modificações que podem ocorrer no produto.

Palavras-chave: medicamentos, denúncias, ineficácia terapêutica.

\section{Abstract}

The administration of ineffective medications can have serious consequences for the health of the patient, and in the case of medicines used to treat life-threatening illnesses can be life-threatening. This study aimed to evaluate the complaints with suspected therapeutic inefficacy of drugs in the samples with satisfactory results analyzed at the National Institute of Quality Control in Health in the period from January 2000 to December 2016 to ascertain the possible sources of harm public health. Of the total 1974 samples of medicinal products from complaints, 424 (21\%) were identified with suspected therapeutic inefficacy, $318(75 \%)$ with satisfactory analytical results. Among these drugs satisfactory, 35 (11\%) were referring to the reference drugs, 68 (21\%) to generic and $215(68 \%)$ to similar. The drugs that have the highest notifications, but satisfactory results were captopril, enalapril maleate, carbamazepine and bupivacaine hydrochloride. All appear in the literature as drugs that may present polymorphs, moreover, captopril, enalapril maleate and bupivacaine hydrochloride are known to be chiral molecules. However, the analytical tests recommended in Pharmacopoeias are not always sufficient to identify possible modifications that may occur in the product.

Keywords: Drugs. Denunciations. Therapeutic inefficacy. 


\section{Introdução}

A administração de medicamentos ineficazes pode trazer consequências graves para a saúde do paciente, e no caso de medicamentos utilizados para tratar doenças potencialmente fatais pode causar um agravamento da doença ou até mesmo colocar a vida em risco. A qualidade de um medicamento significa a garantia de que o mesmo sempre se apresentará seguro e eficaz em consonância com as evidências constantes nos Compêndios Oficiais (Farmacopeias) e nos dados apresentados às autoridades quando de seu registro. Um desvio de qualidade pode significar uma perda de eficácia ou de segurança, expondo o paciente a riscos à saúde. Essa garantia da qualidade se obtém como resultado da consideração de todos os fatores que entra na concepção, desenvolvimento, produção, distribuição e uso do fármaco. A possibilidade de ocorrer modificações no medicamento acabado e, consequentemente, prejudicar a qualidade, mesmo dentro de seu prazo de validade, deve ser levada em consideração e estudada pelo fabricante do medicamento. Faz-se importante, então, controlar a qualidade do medicamento para garantir suas características de maneira uniforme a cada lote de produção (COSTA, 2005).

O controle de qualidade no medicamento propriamente dito é um processo bastante complexo pela própria natureza das amostras. Para que se possa ter confiabilidade nos resultados de uma análise, várias considerações devem ser feitas em relação à amostra e ao método empregado. Em relação à amostra, devem ser considerados alguns parâmetros preconizados nas Farmacopeias como: conteúdo do princípio ativo dentro dos limites experimentais, uniformidade de conteúdo em cada dose, ausência de contaminantes, manutenção da potência, eficácia terapêutica e aspecto até o momento do uso (SANTORO, 1988). Em relação ao método empregado na análise, as especificações da qualidade dos produtos farmacêuticos e de todos os insumos utilizados na fabricação de formas farmacêuticas, é competência exclusiva das farmacopeias (BITTENCOURT, 1997).

Essas Farmacopeias devem incorporar o padrão mínimo de qualidade para a aceitação do produto tendo em vista as tecnologias de produção em uso no país. A utilização desses requisitos farmacopeicos pelo produtor não é obrigatória e sim indicação de requisitos de qualidade cujo cumprimento poderá ser avaliado oficialmente no ato do registro ou quando for submetido a controles laboratoriais pelos órgãos 
governamentais em qualquer momento do prazo de validade do produto (ROSENBERG \& SILVA, 1997).

Apesar das especificações da qualidade dos produtos farmacêuticos serem de competência dos Compêndios Oficiais, o cumprimento integral desses requisitos nem sempre são suficientes do ponto de vista da segurança do consumidor, visto que as especificações farmacopeicas não dizem respeito às boas práticas de fabricação, aos ensaios pré-clínicos e clínicos e aos ensaios de estabilidade (ROSENBERG \& SILVA, 1997).

Medicamentos contendo o mesmo fármaco, na mesma concentração e forma farmacêutica, não necessariamente apresentam o efeito terapêutico na mesma intensidade. Alterações na rota de síntese podem provocar, por exemplo, mudanças nas características físico-químicas do fármaco, podendo ter influência significativa na biodisponibilidade do princípio ativo, comprometendo a eficácia do medicamento (ZHANG et al., 2004; BUENO, 2005).

Os fármacos devem ser liberados da forma farmacêutica na quantidade apropriada e de modo que o início e a duração de sua ação sejam os desejados. As características inerentes ao fármaco, a presença de excipientes que favoreçam ou dificultam a dissolução e as técnicas de fabricação empregadas devem ser bem desenvolvidas, estabelecidas e controladas. Sendo assim, estes fatores necessitam ser exaustivamente estudados durante o desenvolvimento farmacotécnico do produto (ANSEL, POPOVICH E ALLEN, 2013).

Características de uma substância farmacêutica, tais como, polimorfismo e a existência de enantiômeros também podem afetar a segurança do produto, sua eficácia e sua estabilidade, portanto devem ser consideradas no desenvolvimento do produto (ANSEL, POPOVICH E ALLEN, 2013; GASPAROTTO, 2005).

É importante ressaltar que os Compêndios Oficiais, apesar de mencionarem o polimorfismo como característica de algumas matérias-primas em suas monografias, não indicam procedimentos específicos para verificação desses polimorfos como parte integrante dos testes de identificação do produto. Assim, prevalecerão os conhecimentos científicos sobre o assunto, especialmente no caso de fármacos com polimorfos já confirmados pela literatura e que apresentam diferenças na solubilidade das diferentes formas polimórficas, particularmente quando o fármaco é pouco solúvel (CAPUCHO et al., 2008; BRITISH, 2009; ICH, 1999). Analogamente, o mesmo ocorre com fármacos 
que podem apresentar, pelas suas estruturas químicas, características quirais (NOEL et al., 2004; RENTSCH, 2002).

No Brasil, desde 1999, o controle de qualidade sob regime da Vigilância Sanitária é feito pela Agência Nacional de Vigilância Sanitária (Anvisa) e pelos Órgãos de Vigilância Municipais e Estaduais em conjunto com os laboratórios oficiais: Instituto Nacional de Controle de Qualidade em Saúde (INCQS) e os Laboratórios Centrais de Saúde Pública dos Estados, que atuam como fonte geradora de informações capaz de desencadear ações de vigilância sanitária (BRASIL, 1999; SILVA, 2000).

Os Laboratórios Oficiais têm como principal função, a avaliação analítica para fornecer subsídios e elucidar dúvidas quanto à qualidade mínima dos produtos sujeitos à vigilância sanitária. As análises realizadas por estes laboratórios devem estar sempre de acordo com as normas descritas nos Compêndios Oficiais (RIO, 2009).

Este trabalho teve como objetivo avaliar os principais motivos que medicamentos com denúncia de ineficácia terapêutica obtiveram resultados analíticos satisfatórios quando foram analisados pelo INCQS. E o Laboratório de Medicamentos do INCQS utiliza como referência as monografias descritas nos Compêndios Oficiais para a realização dos ensaios analíticos. Sabe-se que existem diversos fatores inerentes à estrutura química do fármaco, relacionados a rota de síntese e características farmacológicas que podem interferir na eficácia do medicamento. Os ensaios farmacopeicos muitas vezes não são suficientes para determinar a fonte de agravo, impossibilitando assim uma avaliação adequada no produto final. A não determinação da fonte de agravo, já que os resultados analíticos foram satisfatórios, acarreta na diminuição da eficiência da ação de vigilância sanitária, tanto na proteção como na promoção da saúde da população (RIO, 2009).

\section{Metodologia}

Foi realizado um estudo retrospectivo no período de janeiro de 2000 a dezembro de 2016, através do software de gerenciamento de amostras laboratoriais (Harpya ${ }^{\circledR}$, versão 2.1.2478, (C) 2015 Harpya Limited), de medicamentos analisados pelo INCQS com denúncia de ineficácia terapêutica. Para este estudo foram utilizadas como referência, as monografias oficiais inscritas na Farmacopeia Brasileira ou na ausência 
desta, monografias oficiais, última edição, de um dos compêndios internacionais conforme RDC n³ 37/2009.

\section{LEVANTAMENTO DOS DADOS}

Foram selecionadas todas as notificações do Programa de Medicamentos provenientes de modalidade fiscal compreendidas no período em estudo e foram consultados no software Harpya ${ }^{\circledR}$ os seguintes itens: ano, número da amostra, data de recebimento, nome do produto, motivo da apreensão e avaliação final (resultado satisfatório ou insatisfatório).

Das amostras provenientes de denúncias, foram selecionadas aquelas com denúncia de suspeita de ineficácia terapêutica e identificadas quanto à avaliação final, se obtiveram resultados satisfatórios ou insatisfatórios. Para as amostras que apresentaram avaliação final satisfatória, foram identificados os princípios ativos e o tipo de medicamento (referência, genérico ou similar).

Dentre os princípios ativos que apresentaram maior incidência de denúncia com suspeita de ineficácia terapêutica, foi realizado revisão de literatura, observando a pertinência de informações sobre polimorfismo e/ou quiralidade desses fármacos. Foram consultadas as bases de dados: PubMed, ScienceDirect, Scielo e ChemWeb.

Baseado nas exigências das legislações vigentes no período retrospectivo avaliado sobre o registro de medicamentos genérico e similar e considerando que dentre os principais fatores que podem interferir na biodisponibilidade dos medicamentos estão o polimorfismo e a quiralidade, estes foram os itens escolhidos para avaliação neste estudo (BRASIL, 2007a,b).

\section{Resultados e Discussões}

Do total de 2992 amostras de medicamentos analisadas no período estudado, 1974 amostras foram provenientes de denúncias, o que corresponde a 66\% do total analisado pelo Setor de Medicamentos do INCQS. Destas 1974 amostras, 424 foram identificadas com suspeita de ineficácia terapêutica, o que corresponde a $21 \%$ do total de denúncias. 
São vários os fatores de grande complexidade relacionados às características físico-químicas dos fármacos, características dos excipientes e alterações na tecnologia de fabricação que, se não estiverem bem controlados durante o desenvolvimento do produto, podem ter influência significativa na biodisponibilidade do princípio ativo, comprometendo a eficácia do medicamento (ANSEL, POPOVICH E ALLEN, 2013). Isso pode ser percebido no grande número de amostras recebidas pelo INCQS com suspeita de ineficácia terapêutica.

Dentre as 424 amostras de denúncias com suspeita de ineficácia terapêutica, 318 foram identificadas com avaliação final satisfatória, o que corresponde a $75 \%$ do total e apenas 106 amostras tiveram avaliação final insatisfatória, o que corresponde a 25\%. Esse alto número de amostras provenientes de denúncias com suspeita de ineficácia terapêutica que apresentaram avaliação final satisfatória (318 amostras) pode estar relacionado, principalmente, ao fato dos ensaios constantes nas farmacopeias não serem suficientes para determinar a fonte de agravo. As normas vêm sofrendo sucessivas revisões com o intuito de acompanhar o conhecimento científico do risco e o desenvolvimento tecnológico da produção. Entretanto, percebe-se uma defasagem muito grande entre as descobertas científicas e a elaboração de normas oficiais, que só absorvem o conhecimento muito tempo depois. A velocidade de introdução de novos produtos, novos coadjuvantes ou novas tecnologias supera os conhecimentos acumulados e consolidados em atos sanitários. Desta forma, novas fontes de agravo à saúde precisam ser determinadas e monitoradas, necessitando de normas específicas (SILVA, 2000).

Das 318 amostras com avaliação final satisfatória, observou-se que 11\% (35 amostras) referia-se a medicamento referência, 21\% (68 amostras) a medicamentos genéricos e 68\% (215 amostras) a medicamentos similares (Tabela 1). O grande número de medicamentos similares pode ser explicado por apresentarem, geralmente, o menor preço e, consequentemente, serem mais adquiridos, principalmente por hospitais da rede pública que compram grandes quantidades através de processos de licitação (BRASIL, 1993).

Em relação ao menor preço comparado aos medicamentos genéricos pode ser pelo motivo de que nem todos os medicamentos similares, no período de janeiro de 2000 a dezembro de 2016, apresentavam estudos de biodisponibilidade relativa no momento do registro, estudos que aumentam o custo do medicamento e que acaba sendo repassado para o consumidor (CAPUCHO et al., 2008). Mas a partir de 2003, 
com a publicação da RDC n 133/2003 (revogada pela RDC n 60/2014), os medicamentos similares começaram a apresentar tal estudo, e por isso, o que estava disponível no mercado, durante o período em estudo, eram medicamentos similares que apresentavam e que não apresentavam estudos de biodisponibilidade relativa. Estima-se que somente a partir de 2013 todos os medicamentos similares comercializados no Brasil passaram a apresentar tais estudos que são essenciais para a garantia de sua qualidade, segurança e eficácia (BRASIL, 2003; BRASIL 2014).

Tabela 1 - Tipo de medicamento de amostras com suspeita de ineficácia terapêutica analisadas pelo INCQS com avaliação final satisfatória no período de janeiro de 2000 a dezembro de 2016

\begin{tabular}{lcc}
\multicolumn{1}{c}{ Tipo de medicamento } & Número de amostras de medicamentos & $\%$ \\
\hline Referência & 35 & 11 \\
Genérico & 68 & 21 \\
Similar & 215 & 68 \\
Total & 318 & 100 \\
\hline Fonte: dados obtidos no Sistama Harpya &
\end{tabular}

A tabela 2 relaciona os fármacos com maiores notificações de suspeita de ineficácia terapêutica analisados no período de janeiro de 2000 a dezembro de 2016 e que apresentaram resultados satisfatórios. Dentre estes fármacos, os de maiores incidências de registros foram captopril, maleato de enalapril, carbamazepina e cloridrato de bupivacaína. Todos estes constam na literatura como fármacos que podem apresentar polimorfos ou pseudopolimorfos. Além disso, captopril, maleato de enalapril, e cloridrato de bupivacaína são substâncias quirais (BRANDÃO, 2006; COELHO, 2001; NAIR, et al., 2002; GIRON, 1995; ARAUJO, 2012).

Tanto para o medicamento genérico quanto para o similar, o peticionário do registro deve apresentar os principais contaminantes do(s) fármaco(s), seus limites de quantificação, além dos teores de estereoisômeros e determinação dos polimorfos, quando aplicável. No momento do registro do medicamento, a legislação não limita quantos fabricantes do fármaco que o peticionário pode apresentar. No entanto, os estudos de equivalência farmacêutica e bioequivalência/biodisponibilidade relativa são realizados apenas com um fabricante do fármaco do medicamento (NOEL, et al., 2004; BRASIL, 2014; CREMASCO, 2013). 
Tabela 2 - Medicamentos com denúncia de ineficácia terapêutica analisados no período de 2000 a 2016 que apresentaram resultados satisfatórios

\begin{tabular}{lcc}
\multicolumn{1}{c}{ Princípio ativo } & $\begin{array}{c}\text { Número de amostras } \\
\text { analisadas* }\end{array}$ & Classe terapêutica** \\
\hline Ácido valpróico & 6 & anticonvulsivante \\
Amoxicilina & 5 & antibiótico \\
Captopril & 38 & anti-hipertensivo \\
Carbamazepina & 15 & anti-convulsivante \\
Cefalexina & 6 & antibiótico \\
Ceftriaxona Dissódica & 8 & antibiótico \\
Cloridrato de Bupivacaína & 16 & anestésico local \\
Cloridrato de Bupivacaína + Glicose & 19 & anestésico local \\
Cloridrato de Vancomicina & 7 & antibiótico \\
Dipirona + Mucato de Isometepteno & 6 & analgésico associação \\
+ Cafeína & & \\
Etinilestradiol + Levonogestrel & 12 & contraceptivo oral \\
Heparina Sódica & 6 & anticoagulante \\
Maleato de enalapril & 16 & anti-hipertensivo \\
Maleato de Midazolam & 8 & benzodiazepínico \\
\hline Fonte: dados obtidos no Sistama Harpya ${ }^{*}$ & & \\
$*$ THE MERCK INDEX, 2013; MARTINDALE, 2014. & &
\end{tabular}

O perfil de dissolução faz parte da documentação complementar exigida para os medicamentos, no caso da apresentação de mais de um fabricante do(s) fármaco(s), que não foram submetidos aos estudos de Equivalência Farmacêutica e/ou Biodisponibilidade Relativa/Bioequivalência, conforme preconiza as legislações vigentes (BRASIL, 2007a,b; BRASIL, 2014). Porém, segundo Gasparotto (2005), este ensaio não é suficiente para prever uma possível alteração na biodisponibilidade, considerando que o(s) fármaco(s) pode $(\mathrm{m})$ apresentar diferentes isômeros e/ou diferentes polimorfos que não são adequadamente identificados pelos ensaios farmacopeicos atuais.

Assim como os estudos da matéria-prima são importantes para se obter um medicamento eficaz e seguro, são também importantes os estudos sobre os diferentes isômeros e o comportamento polimórfico dos fármacos no produto acabado. Além da rota de síntese, podem ocorrer alterações físico-químicas no fármaco durante o processo 
de produção e armazenamento do medicamento que consequentemente poderão interferir na qualidade do produto acabado (VIPPAGUNTA et al., 2001; ANSEL, POPOVICH E ALLEN, 2013). Entretanto, os ensaios presentes nas farmacopeias nem sempre são suficientes para identificar possíveis modificações que podem ocorrer com o fármaco, impossibilitando, muitas vezes uma avaliação adequada no produto final (RIO, 2009).

\section{Conclusões}

No período em estudo, 1974 amostras de medicamentos (66\%) foram provenientes de denúncias, sendo 424 (21\%) identificadas com suspeita de ineficácia terapêutica. Destas, 318 amostras (75\%) obtiveram avaliação final satisfatória. Dentre estas, os quatro princípios ativos que apresentaram maior incidência foram captopril, maleato de enalapril, carbamazepina e cloridrato de bupivacaína. Todos constam na literatura como fármacos que podem apresentar polimorfos ou pseudopolimorfos. Além disso, captopril, maleato de enalapril e cloridrato de bupivacaína apresentam quiralidade.

No Relatório de Produção para registro de medicamentos genérico e similar junto a Anvisa, o peticionário do registro deve apresentar informações sobre provável influência de polimorfos e proporções de estereoisômeros do fármaco. Mas em nenhum momento, a legislação define claramente os critérios de análise que devem ser seguidos. Percebe-se que há de se aperfeiçoar os mecanismos de regulação de modo a prevenir riscos à saúde da população.

Os ensaios presentes nas farmacopeias nem sempre consideram as possíveis modificações que podem ocorrer com o fármaco, impossibilitando uma avaliação adequada no produto final. Assim, faz-se necessário o desenvolvimento de métodos analíticos para a avaliação dos teores dos estereoisômeros e determinação de prováveis polimorfos para fármacos em que estas características possam comprometer a eficácia e segurança do produto.

\section{Agradecimentos}

Ao INCQS/FIOCRUZ pelo incentivo à realização deste trabalho. 


\section{Referências}

ANSEL HC; POPOVICH NG; ALLEN LV. Formas Farmacêuticas e Sistemas de Liberação de fármacos. 9a ed. São Paulo: ArtMed, 2013.

ARAUJO GLB; JUNIOR AP; ANTÔNIO SG; SANTOS COP; MATOS, JR. Polimorfismo na produção de medicamentos. Revista de Ciências Farmacêuticas Básica e Aplicada, v. 33, n. 1, p. 27-36, 2012.

BITTENCOURT, CF. O Desenvolvimento da Farmacopéia Brasileira IV. In: BONFIM, J.R.A.; MERCUCCI, V.L., org. A construção da politica de medicamentos. São Paulo: Hucitec, 1997.p. 241-250.

BRANDÃO ALA. Influência do polimorfismo na farmacotécnica de cápsulas no setor magistral. Revista Racine, v. 91, p. 01-28, 2006.

BRASIL. Lei no 9.782, de 26 de janeiro de 1999. Define o Sistema Nacional de Vigilância Sanitária, cria a Agência Nacional de Vigilância Sanitária, e dá outras providências. Diário Oficial da União, 27 de janeiro de 1999, seção 1.

BRASIL. Resolução RDC no 16, de 02 de março de 2007. Aprova o Regulamento Técnico para Medicamentos Genéricos. Diário Oficial da União, 05 de março 2007a, Seção 1 .

BRASIL. Resolução RDC no 17, de 02 de março de 2007. Dispõe sobre o registro de Medicamento Similar. Diário Oficial da União, 05 de março 2007b, Seção 1.

BRASIL. Lei no 8.666, de 21 de junho de 1993. Regulamenta o art. 37, inciso XXI, da Constituição Federal, institui normas para licitações e contratos da Administração Pública e dá outras providências. Diário Oficial da União, 21 de junho 1993, Seção 1.

BRASIL. Resolução RDC no 37, de 06 de julho de 2009. Trata da admissibilidade das Farmacopeias estrangeiras. Diário Oficial da União, 08 de julho de 2009, Seção 1.

BRASIL. Resolução RDC no 133, de 29 de maio de 2003. Dispõe sobre o registro de Medicamento Similar. Diário Oficial da União, 02 de junho 2003, Seção 1. 
BRASIL. Resolução RDC no 60, de 10 de outubro de 2014. Dispõe sobre os critérios para a concessão e renovação do registro de medicamentos com princípios ativos sintéticos e semissintéticos, classificados como novos, genéricos e similares, e dá outras providências. Diário Oficial da União, 13 de outubro 2014, Seção 1.

BRITISH PHARMACOPEIA 2009. London. The Stationery Office, 2008. 1 CDROM.

BUENO MM. Implantação, evolução, aspectos técnicos e perspectivas da regulamentação técnica de biodisponibilidade relativa e bioequivalência de medicamentos genéricos e similares no Brasil. 2005. Dissertação (Mestrado em produção e Controle Farmacêuticos) Faculdade de Ciências Farmacêuticas, Universidade de São Paulo, 2005.

CAPUCHO, HC; MASTROIANNI, PC; CUFFINI, S. Farmacovigilância no Brasil: a relação entre polimorfismo de fármacos, efetividade e segurança dos medicamentos. Revista de Ciências Farmacêuticas Básica Aplicada, v. 29, n. 3, p. 277- 283, 2008.

COELHO, FAS. Fármacos e Quiralidade. Cadernos temáticos de Quimica Norva na Escola, n.3, 2001.

COSTA L. Avaliação do polimorfismo de fármacos utilizados para a produção de medicamentos genéricos no Brasil. 2005. 93 f. Dissertação (Mestrado em Ciências Farmacêuticas) - Universidade Federal do Rio Grande do Sul, Porto Alegre, 2005.

CREMASCO, MA. A fronteira da indústria farmacêutica no Brasil: enantiômeros. Ciência e Cultura, v.65, n.3, p. 4-5, 2013.

GASPAROTTO FS. Fatores relacionados à sintese de matérias-primas que podem alterar a biodisponibilidade do medicamento genérico. 2005. 87 f. Dissertação (Mestrado em Ciências Farmacêuticas) - Universidade Federal do Rio Grande do Sul, Porto Alegre, 2005.

GIRON D. Thermal analysis and calorimetric methods in the characterisation of polymorphs and solvates. Thermochimica Acta, v. 248, p. 1-59, 1995.

ICH - International Conference on Harmonisation of Technical Requirements for Registration of Pharmaceuticals for Human Use. Specifications: test procedures e 
acceptance criteria for new drug substances and new drug products: chemical substances - Q6A, 1999.35p. Disponível em: < www.ich.org >. Acesso em: 27 out. 2017.

MARTINDALE. The complete drug reference. Editor: S. C. Sweetman. 38. ed. London: Pharmaceutical Press, 2014. 2v.

NAIR R; GONEN S; HOAG SW. Influence of polyethylene glycol and povidone on the polymorphic transformation and solubility of carbamazepine. International Journal of Pharmaceutics, v. 240, p. 11-22, 2002.

NOËL, F; VOLPATO N; RIBEIRO A; BOLUTARI E; CUPELLO T. Estudos de bioequivalência para fármacos que apresentam estereoisomerismo. Infarma-Ciências Farmacêuticas, v. 16, 2004.

RENTSCH KM. The importance of stereoselective determination of drugs in the clinical laboratory. Journal of Biochemical and Biophysical Methods, v. 54, p 1-9, 2002.

RIO AS. Avaliação das denúncias de ineficácia terapêutica de medicamentos com resultados satisfatórios realizados no INCQS no periodo de janeiro de 2000 a dezembro de 2008. 2009. 64 f. Monografia (Especialização em Controle de Qualidade de Produtos, Ambientes e Serviços Vinculados à Vigilância Sanitária) - Instituto Nacional de Controle de Qualidade, Rio de Janeiro, 2009.

ROSENBERG FJ; SILVA ABM. As Farmacopeias e o Laboratório Oficial de Controle de Qualidade. In: BONFIM, JRA; MERCUCCI, VL, organizador. $A$ Construção da Politica de Medicamentos. São Paulo: Editora Hucitec; 1997. p. 251-255.

SANTORO, MIR. Controle integral de medicamentos. In: Introdução ao Controle de Qualidade de Medicamentos. São Paulo: Atheneu, 1988. p. 6-12.

SILVA ACP. O laboratório Oficial na avaliação analítica. In: ROSENFELD, S, organizador. Fundamentos da Vigilância Sanitária. Rio de Janeiro: Fiocruz; 2000. p. 271300 .

THE MERCK INDEX: an encyclopedia of chemicals, drugs and biologicals. 15 ed. New Jersey: Merck \& Co., 2013. 2708p. 
VIPPAGUNTA, SR; BRITTAIN, HG; GRANT, DJ. Crystalline solids. Advanced Drug Delivery Reviews, v.48, p.3-26, 2001.

ZHANG GG; LAW D; SCHMITT EA; QIU Y. Phase transformation considerations during process development and manufacture of solid oral dosage forms. Advanced Drug Delivery Reviews, v. 56, p. 371-90, 2004. 